\title{
A STUDY OF WAYFINDING IN TAIPEI METRO STATION TRANSFER: MULTI-AGENT SIMULATION APPROACH
}

\author{
Kuo-Chung $\mathrm{WEN}^{1} *$ and Wei-Chen $\mathrm{SHEN}^{2}$ \\ ${ }^{1}$ Associate Professor, Graduate Institute of Architecture and Urban Design, Chinese Culture University, Taiwan \\ ${ }^{2}$ Postgraduate Student, Graduate Institute of Architecture and Urban Design, \\ Chinese Culture University, Taiwan \\ *Corresponding author (wenkc@faculty.pccu.edu.tw)
}

\begin{abstract}
Mass Rapid Transit (MRT) station is the transport node, with population concentrated, closed-space, more gates this type of space a high risk of accidents hidden. The study based multi-agent simulation (MAS) system to create wayfinding simulation in MRT station, to create an effective evacuation simulation model. It can use to this type of space as a basis for risk assessment and future planning and design of the test platform.
\end{abstract}

Keywords: Multi-agent Simulation, MRT Station, Wayfinding, Spatial Information Technology

\section{Introduction}

Transfer type is designed to enhance the metro station with the city transportation system links. This is the rail transport system as a very important part. Outbound passengers do not need the case to be different routes within the station interchange. But this spatial pattern of increased cognitive is difficulty of space travelers. Space is huge scope to become space tourists find its way wide.

Space function intensive information processing is increase the amount of passenger wayfinding. Spatial three-dimensional flow of visitors increased the difficulty of positioning the vertical direction. This type of space in terms of passengers for the first visit, places to instruct passengers to the direction of the most important indicators of wayfinding system.

Wayfinding is to seek answers to questions of space, that is, to solve the problem of space. More precisely, find its way through the cognitive abilities of spatial information received will be absorbed by the development of wayfinding information as the basis for planning and decision-making, then these decisions into action.

This study of space finds its way as the spindle. We take spatial information technology in GIS to build the simulation environment, apply mutil-agent simulation method to simulate passengers on the MRT station in wayfinding. Its main purpose is to understand the existing space station for the effectiveness of passenger wayfinding.

\section{Pedestrian movement simulation}

Pedestrian movement model is the real world characteristics of pedestrian behavior and mathematical description of the abstract[1] despite the person's behavior is more complex, sometimes more or less manifested in disorder, but it is still can be found Regularity.

As an analytical tool, pedestrian movement simulation techniques can be applied to public safety areas, such as ships, aircraft and other large transportation design, sports stadiums, subway stations and other large public facilities, design and so on.

\subsection{Multi-agent Systems, MAS}


Multi-agent system (MAS) can also be extended to Multi-Agent Based Modeling (MABM), also known as multi-agent model. The main basis of the actors combined mode and the grid pattern to produce dynamic simulation scenarios. Multi-agent system can be defined in a common environment, the interaction of a group of actors, actors have to fix themselves and their environment.[2]

MAS have the ability to solve some of their own problems, and to study the interaction between all the targets. Interaction may occur in between actors and actors, or actors and in between their environment. Actors in the MAS may be self-interested or cooperative, the computer based on the actors themselves attribute to establish a basis model.

\subsection{NetLogo}

NetLogo is a freely downloadable, agent-based software package that was created at the Center for Connected Learning and Computer-Based Modeling (CCL) at Northwestern University, directed by Uri Wilensky. It is the latest in a series of multi-agent simulation environments that includes StarLogo, which was developed at the MIT Media Lab by Wilensky and Mitchel Resnick. NetLogo illustrates what can happen when populations of turtles are given sets of rules to obey. Despite the entry-level programming interface, NetLogo is capable of quite sophisticated modeling and allows experienced programmers to add their own Java extensions. As a result, NetLogo has been widely used by a broad audience, from elementary school children to academics in the social, computer, and "hard" sciences; and the online community page includes models constructed by a wide range of representatives from each of these segments of the population[3].

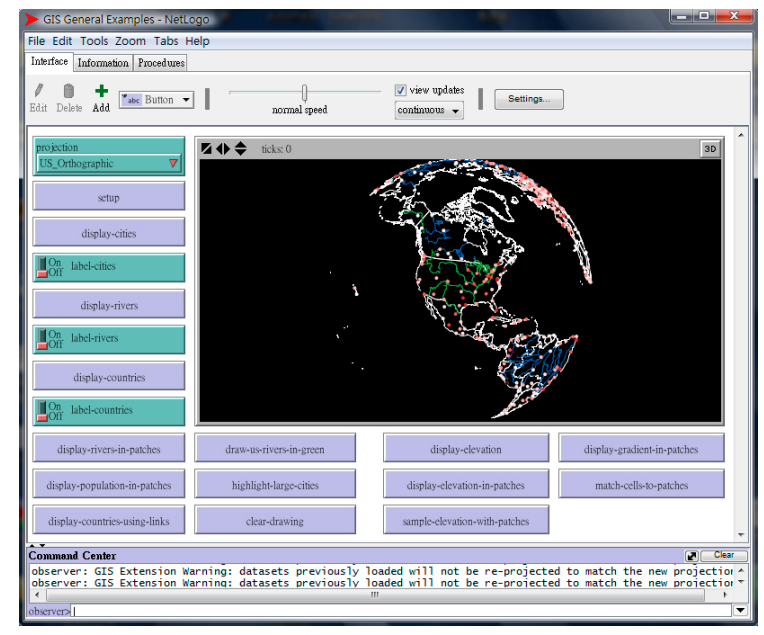

Fig 1 netlogo Interface

\subsection{Wayfinding}

The wayfinding is an interdisciplinary problem. It involves cognitive psychology, linguistics, graphic design, architecture, interior design, landscape architecture, site design, urban design, behavior, facilities management and a series of fields. Wayfinding related to the theory in the field of environmental psychology has many expositions. However, the available information is limited. The other areas involved in the theoretical discussion of this question a lot, but most failed to meet the considerable theoretical depth [4].

In 1960, the urban designer Keven Lynch in his book "Urban Imagery" in the first use of "way-finding" to state the term for a person's understanding and insight into the urban environment, by subjects of the common factors of hand-painted maps identification and analysis, summarized the Space, Structure, Continuity, Visibility, Penetration and the Identity is to avoid the elements lost in the urban space.

\section{Theory}

In this study, the main points and theories are as follows: 3.1

1) Human moving

Pedestrian movement with a clear standard with respect to the road traffic has significantly different characteristics 
with more unbridled. the human moving pedestrians due to the psychological state and purpose was different and appear to be more complex, affecting not only the pavement surrounding the individual actions also affected the traffic flow characteristics of the overall.

2) Time

Time-based an important part of the study, the time for assessing a project can determine the user's movement in the space of efficiency. The study will be of pedestrians to the flow calculate, the time was one of the elements of them.

\section{3) Space}

The space information can help people to understand the space that they are. Arrived at the new environment, Pathfinder will perceive the information to the surrounding environment, choose to collect environmental information, after digestion, in the hearts of cognition, the development of its plan as the basis for wayfinding.

\section{4) Field}

The field can be referred to as the "horizon", people mainly rely on the moving visual information, the use of such sensing means to find the way forward. Spatial information field also includes space to move the "people", the behavior of space users will be affected by these changing information and follow the change.

\section{Simulation}

Operation of this paragraph description of the method

\subsection{Simulation location}

Taipei main station to the system point is divided into the Taiwan Railway, Taiwan High Speed Rail and MRT is composed of three rail transit system; terms of building structure, six stories above ground and underground four-story building. Ground floor main hall, the TRA, HSR ticket booth and passenger seating area; the Taiwan Railway Administration, High Speed Rail platforms are located in the ground floor level; MRT Bannan Line (blue line), Danshui line (red line) of the month Third, respectively, on the ground floor units, four.

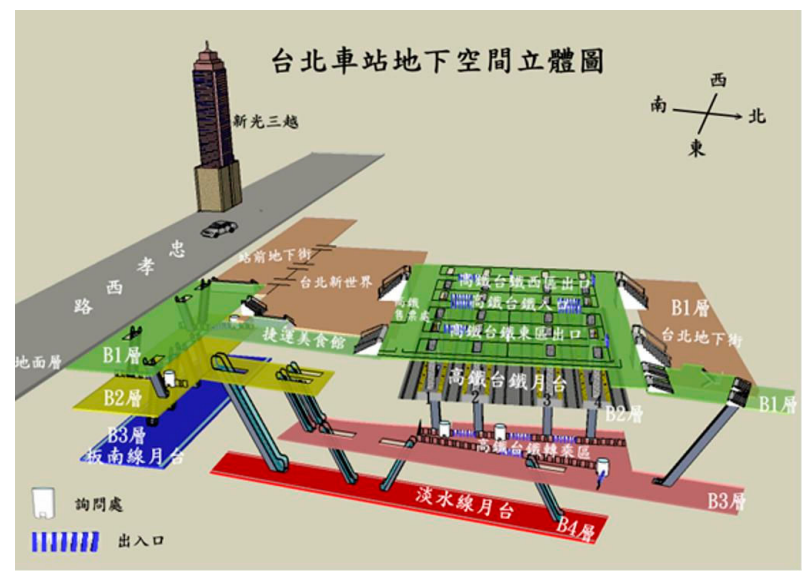

Fig 2 Overview map of Taipei Station [5]

\subsection{Simulation method}

\section{1) Netlogo}

This study, use the Netlogo be a simulation platform, using the features of this software to produce "human" behavior simulation. Netlogo the system can be divided into, actors(turtle), environment(patch), observers (observer), and the actors can be people, vehicles, animals and even city-specific development projects and other unique factors, the independent individual Influence each other to produce different reactions.

\section{2) Concept field}

Being used in the simulation field concept, making the space of each "agent" could generate an observation radius, the radius of this observation to the use of actors to find their own way forward.

\subsection{Simulation process}

In this study, based on the MAS for the spindle system to an agent, beginning from the construction of space to the programming, create a dynamic environment are all based, in order to achieve a complete wayfinding simulation system. By the MAS, space, people, each combination of simulation process: 


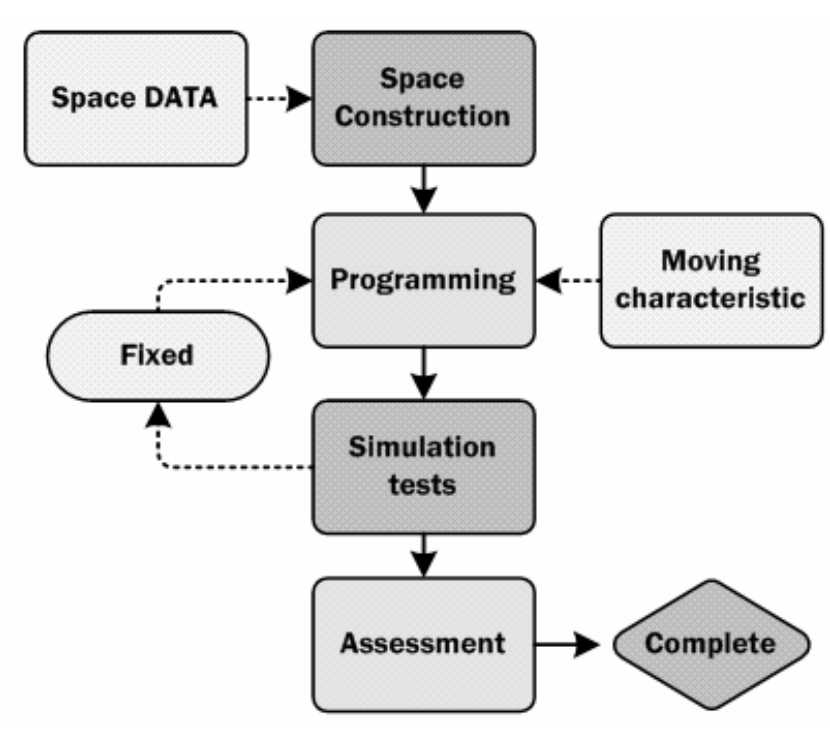

Fig 3 Simulation process

1) Space formed to collect spatial attribute data, constructed the simulation space.

2) Programming, the spatial information and attribute data to write the program into the pedestrian, and establish pedestrian movement patterns.

3) Testing program and constantly modify the wayfinding model

4) The project simulation to simulate the results.

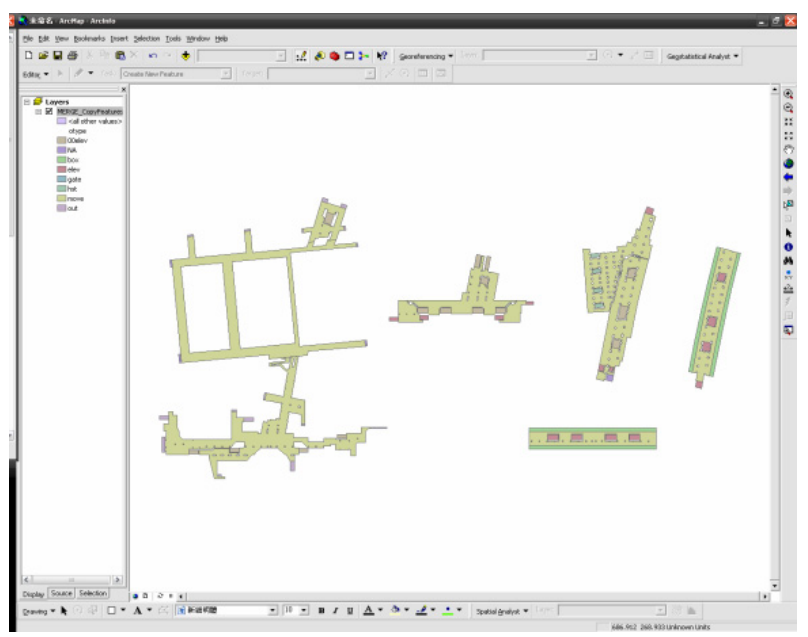

Fig 4 Space Construction

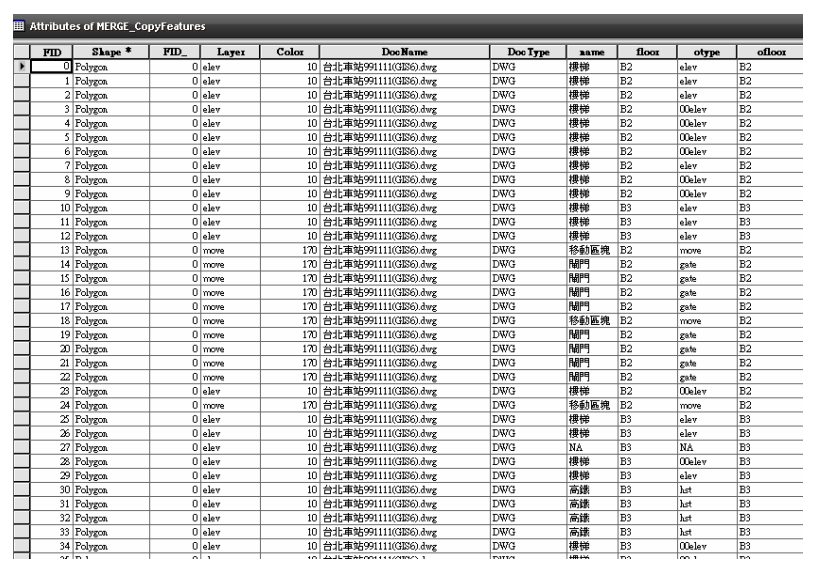

Fig 5 Space Data

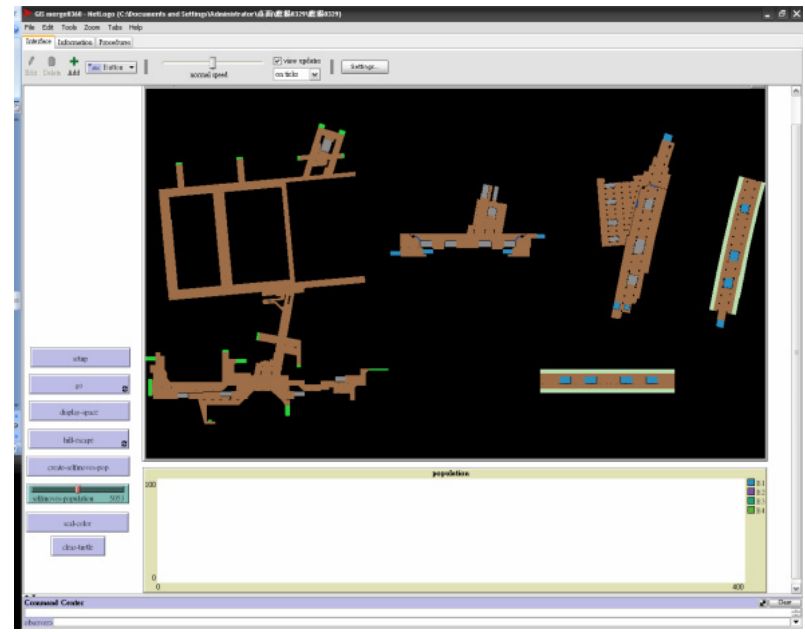

Fig 6 simulation interface

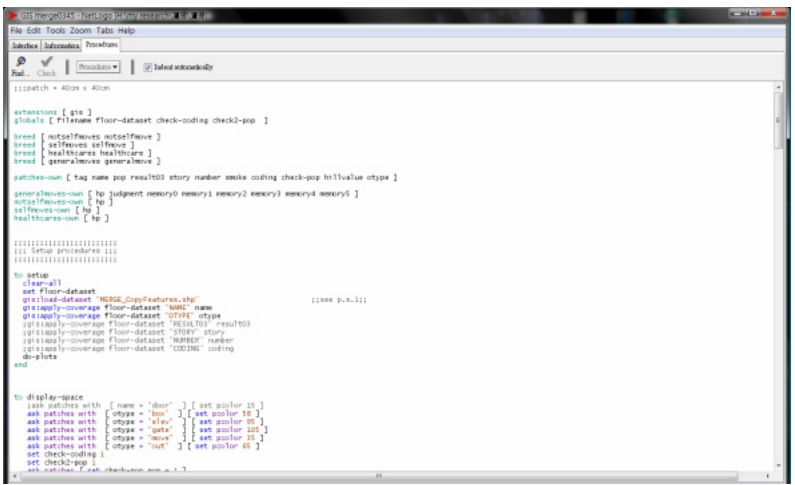

Fig 7 Programming

\section{Results}

Used wayfinding simulation program to operate the evacuation simulation in Taipei main station, it can be seen in order of evacuation operations. Peoples use the field to find the shortest path to the exit, other people follow the first moving person, try to left this space. 


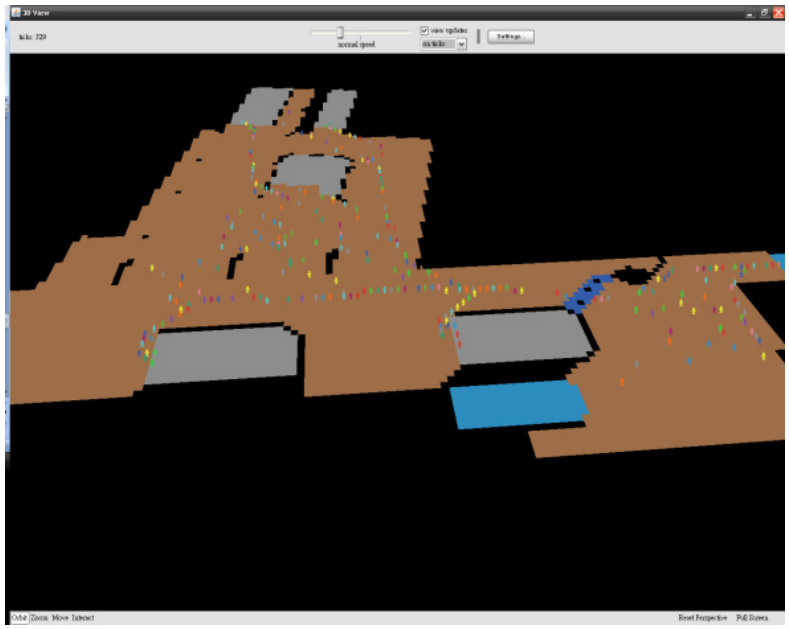

Fig 8 Simulation conditions

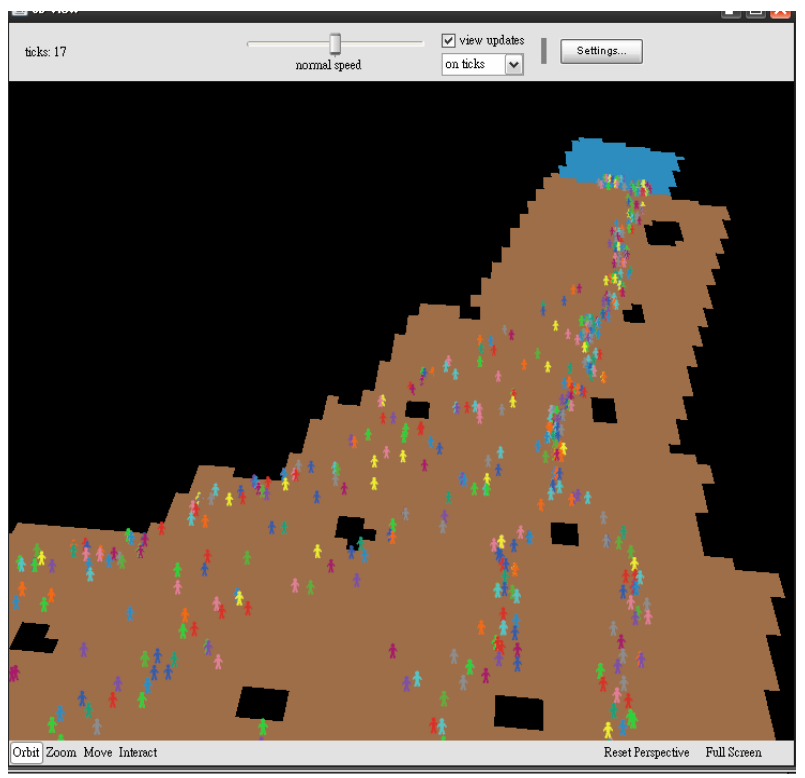

Fig 9 Simulation conditions

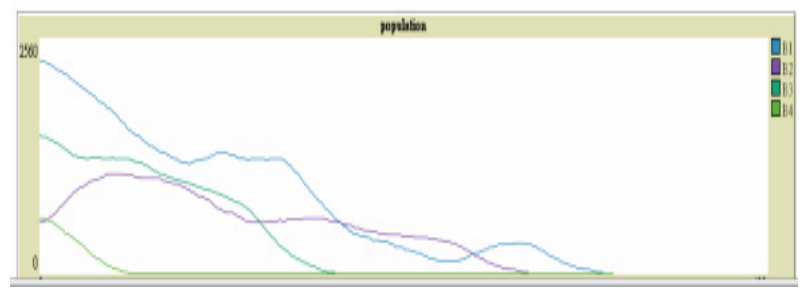

Fig 10 Changes in the total number of each floor plans

As can be seen from Figure 10 when the evacuation project changes in the number of each floor can be learned from this figure the efficiency of the evacuation floors, and with Figure 8 and Figure 9 to observe the movement route compared to the space being crowded Location or causing blockage of the phenomenon.

\section{Conclusion}

In this study, the use of theory and Netlogo MAS simulation platform based on the full agent properties in the MAS system, which is the agent's autonomy and interactivity to successfully build a simulation model for effective wayfinding. The developed system can then add other elements of the simulation for various projects, such as setting targets and disaster prevention systems and other mobile simulation.

\section{References}

[1] Qing-mei Hu, et al., Influence of Exit Layout of a Metro Station on Pedestrian Evacuation, journal of the china railway society, 2009. 31(3): p.

111-115.

[2] Tang, F. and X. Zhang, A GIS-Based $3 D$ Simulation for Occupant Evacuation in a Building. Vol. 13. 2008: Elsevier Science. 58-64.

[3] Sklar, E., NetLogo, a Multi-agent Simulation Environment. Artificial Life, 2007. 13(3): p. 303-311.

[4] Niu Li, Discussion of way-finding under Natural Condition by Simulating the Procedure of Navigation. HuaZhong Architecture, 2005(23) 4: p. 48 .

[5] Chia-Wen Wu, The Research of Wayfinding in the $3 D$ Underground Space of Taipei Main Station, in Department of Geography. 2008, National Changhua University of Education. p. 110. 